\title{
STEAM: education and communication with Art at ATLAS and CMS
}

\author{
Pierluigi Paolucci*1 \\ Istituto Nazionale di Fisica Nucleare di Napoli \\ Complesso Universitario di Monte Sant Angelo Edificio 6, 80100 Napoli, Italy \\ E-mail: Pierluigi.paolucci@na.infn.it
}

\section{Angelos Alexopoulos ${ }^{1}$}

CERN, Switzerland

E-mail: angelos.alexopoulosecern.ch

\section{Michael Hoch ${ }^{1}$}

Austrian Academy of Sciences, Austria

E-mail: michael.hoch@cern.ch

\section{Claire Adam-Bourdarios ${ }^{2}$}

LAL, Univ. Paris-Sud, CNRS/IN2P3, France

E-mail: claire.bourdarios@cern.ch

Recent developments in science education policy and practice suggest that successful learning in the 21 st century requires the horizontal connectedness across areas of knowledge by linking the arts and humanities with science, technology, engineering and mathematics (STEM) subjects. The rapidly increasing STEAM movement calls for arts integration into science teaching and learning to help school students develop skills that are necessary to thrive in an innovation economy. Education and outreach in highenergy physics are not an exception to these developments. This paper describes specific education and outreach initiatives by the ATLAS and CMS collaborations that use a cross-disciplinary approach to engaging the public and especially young people not only with the excitement of scientific research in particle physics but also with its positive technological and social externalities.

\footnotetext{
* speaker

${ }^{1}$ on behalf of CMS collaboration

${ }^{2}$ on behalf of ATLAS collaboration
} 


\section{Introduction}

Recent years have seen a growing consensus in the science education community that STEM (Science, Technology, Engineering and Mathematics) teaching and learning at school and tertiary level can benefit considerably from the introduction of the arts [1] [2]. The so-called STEAM (Science, Technology, Engineering, Arts and Mathematics) movement has also been recognized by science education policy makers as a useful framework for helping young people establish and advance $21^{\text {st }}$ century skills and competencies that are necessary to thrive in an innovation economy [3]. The need to combine art with school science goes, however, beyond calls for employability skills development alone, by emphasizing that art-based activities also "represent one of the best approaches to integrating creativity, imagination and science" [4]. And this is important for it reminds us a widely shared, yet taken-for-granted, view that "science is an activity that involves creativity and imagination as much as many other human activities" [5].

If we accept that "creativity is as important in education as literacy and should be treated with the same status" [6], it is then reasonable to think that a shift from STEM to STEAM may also be of value in physics education research and practice. In this paper, we present an overview of STEAM initiatives undertaken recently by the Outreach and Communications groups of the ATLAS and CMS collaborations respectively. While different in many respects, the initiatives presented in this paper share one common characteristic, that is, the acknowledgement that successful learning in the $21^{\text {st }}$ century depends on "horizontal connectedness across areas of knowledge and subjects as well as to the community and the wider world" [7]. We argue that large research infrastructures, including those in high energy physics, have strong potential in this regard because of their capacity to act as incubators for innovative ideas and projects that can generate positive externalities for society by infusing creativity into their education activities [8].

\section{STEAM activities in ATLAS}

The mandate of the ATLAS Outreach group focuses primarily on the development and support of the experiment's communication platforms and content, educational tools, institutebased outreach programs, local events, and visits. But the group has also developed, over the years, contacts with a wide range of other disciplines: artists, educators, and other innovators of technologies and education methods that can help us to reach new, larger and more diverse audiences.

STEAM in ATLAS comprises projects that are proposed by members of the collaboration, funded by their institutes, and supported by the Outreach group. Each project is first presented to the group with clear definitions of concept, goals, target audiences, messages, potential partners, and responsibilities. Those projects considered feasible are then presented to the collaboration management for approval. If approved, the authors are allowed to use the ATLAS 
branding and are helped by the Outreach group for material, publicity, news, web sites, etc. The two partnerships presented below illustrate this strategy.

\subsection{Projecting pARTicles}

Agnes Chavez is a media artist and STEAM educator in the United States [9], whose goal is to use new media arts to explore, teach, and communicate scientific concepts. Following a two-day immersion into particle physics (e.g. explanation of the Higgs field and its associated boson) and the exploration of an iPad tool [10], students discover projection art as a medium of expression and communication. Together, they storyboard, design, and document a live light projection, inspired by the physics concepts, onto a building.

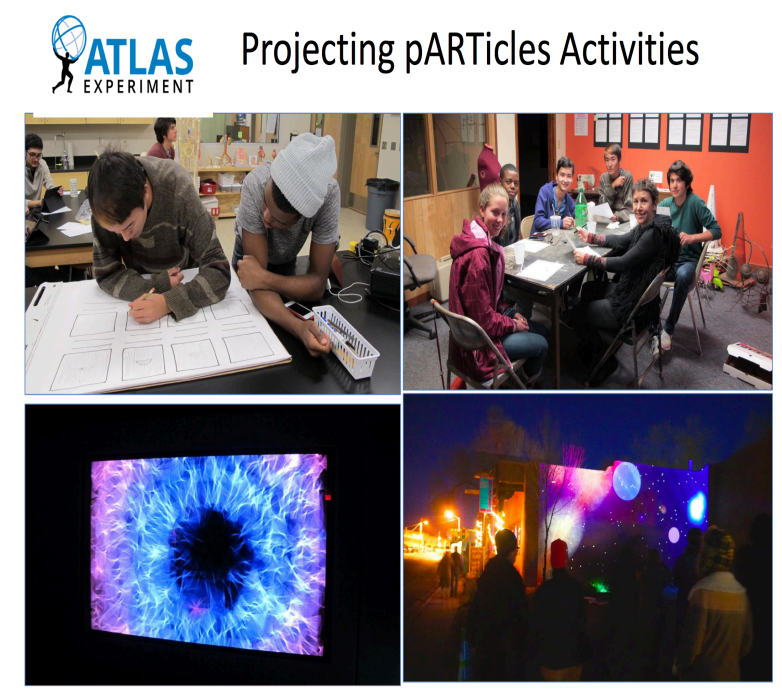

Fig.1: Activities with students during the ATLAS workshop

Informal partnership with the outreach group gradually developed through a series of pilot workshops:

- 2014: The second pilot workshop began with a Virtual Visit [11] to the ATLAS Control Room.

- 2015: During a two-week research stay at CERN, Chavez met a number of ATLAS physicists to discuss and refine concepts.

- 2015: A pilot workshop held in Cuba, included the presence of an ATLAS scientist [12].

- 2016: A three-day workshop at the Harwood Museum (Taos, New Mexico), led by students who participated in a previous workshop, included the integration of an LHC Master Class in Day 1, with support from Quarknet and US ATLAS.

During a second, week-long research visit to CERN in 2016, observations of these pilot workshops were presented: student engagement and understanding increased at each workshop. Invitations to museums and festivals were succeeding to bring HEP concepts to new audiences in an effective manner. It was then proposed that the next steps would involve a stronger connection to nearby laboratories and the development of teaching modules based on various 
HEP concepts. The development team comprises two artists, two ATLAS physicists and a Quarknet educator [13].

\subsection{Quantizer: a new interface for musical expression}

Quantizer is an interdisciplinary tool and platform developed in partnership with the Responsive Environment Group at the well known MIT Media Lab [14]. The project goal is to transform ATLAS data into sound and explore how these could be a source of inspiration for musicians and the general public.

The ATLAS collaboration supports the project through communication and by permitting access to the data stream, which feeds the live event display. Event kinematics and the detector information contained in these events are transformed into musical sequences by a set of tools developed by MIT. A web site [15] developed and maintained by both partners provides outreach information, and invites interested musicians to contact the team to develop their own composition maps.

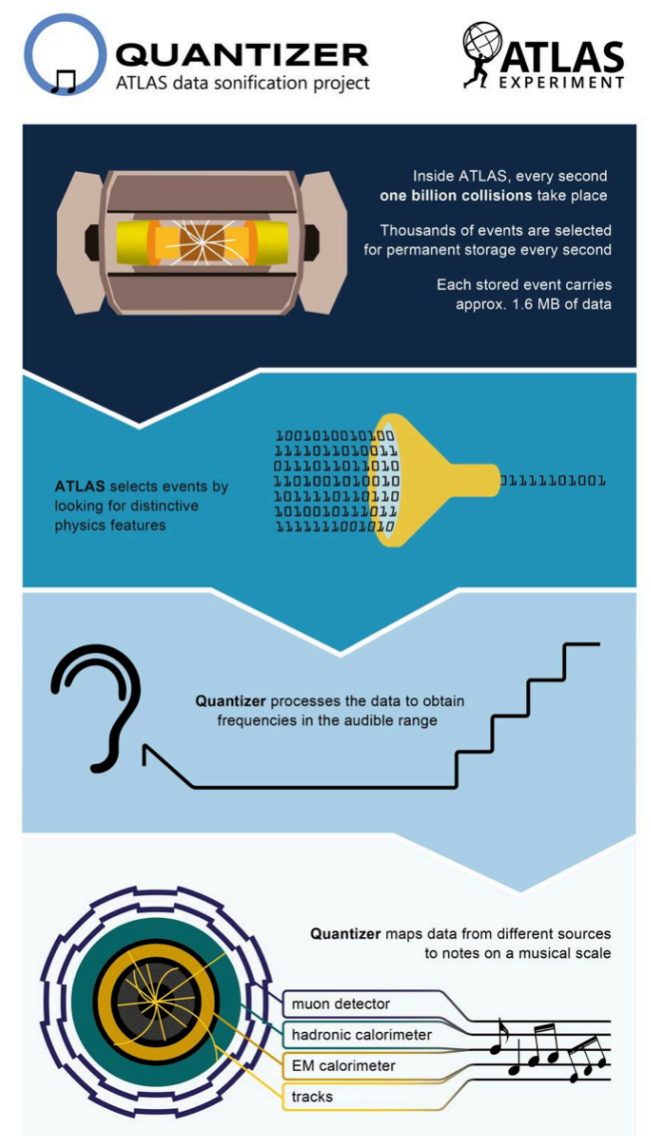

Fig.2: Visual description of the ATLAS Quantizer project.

Fig.2: June 2015: early prototype demonstration in a "Physics of Music and Music of Physics" workshop at the Montreux Jazz festival [16]; 
May 2016: project web site opened to the public, with good coverage in media which usually do not cover HEP. Abstracts for posters accepted at two major conferences: CHI (Computer Human Interface Conference) and NIME (New Interface for Musical Expression) [17].

The team is in touch with various musicians and "sonification" experts. Copyright aspects, long term maintenance, and development of the platform are currently being consolidated. Access to live stream is clearly interesting for this community and for artists who want to setup live events and installations. The development team consists of two ATLAS physicists and two MIT experts of new musical expressions and tools [18].

\section{STEAM activities in CMS}

Over the last few years, the Communications group of the CMS experiment at CERN has developed an integrated framework for education, outreach and communication that revolves around a set of distinct yet complementary programmes and projects, which aim to serve the needs of a diverse network of stakeholders including the CMS institutes, student and teacher communities, and the wider public [19]. From on-site and virtual visits to particle physics Masterclasses and open data for the public, these activities, to name a few, are designed to act as engagement mechanisms allowing multiple audiences to have authentic experiences with the science, engineering and technology that drive research and discovery in high-energy physics.

STEAM in CMS is situated principally within art@CMS [20][21] an education and outreach programme that was founded in 2012 by Austrian particle physicist, photographer and artist Michael Hoch. As evidenced in his recently published photographic work [22], Hoch introduced the idea that the inherent beauty and symmetry of the CMS detector can evoke wonder, curiosity and interest in particle physics by the public and especially by young people. With the support of the CMS Collaboration, and more recently of the CREATIONS EU project [23], art@CMS has evolved over the last three years into a dynamic network of collaborations involving CMS scientists, individual artists, art institutes and cultural institutions, nonprofit arts groups, and students and educators in more than twelve countries. The programme is presented in more detail below.

\section{1art@CMS}

In response to the need to build more bridges between the CMS scientific community and the public, art@CMS was originally developed as a vehicle for public engagement with particle physics through the arts and mainly through the organization of visual art exhibitions. Two lifesize images of the CMS detector that were installed in 2011 and 2012 at CERN became the departure point for a series of photographs and photographic collages created by Michael Hoch. This set of work was then exhibited in CERN and Vienna in 2012 and early 2013 in the framework of scientific conferences and public events, with the aim to enact a dialogue between the worlds of particle physics, education and the arts. Over the following three years, art@CMS 
has evolved into a solid education and outreach programme with two complementary modules: exhibitions for the general public, and SciArt workshops for secondary school and third-level students. As Figure 1 shows, as of mid-2016 almost 145,000 people have visited 42 art@CMS exhibitions organized in 25 cities across 17 countries in four continents. These are often combined with LHC-related events such as public talks, conferences and science festivals with the participation of the local communities, serving as forums for dialogue on the role of science in society. For the purposes of this paper, we will focus on the SciArt workshops only.

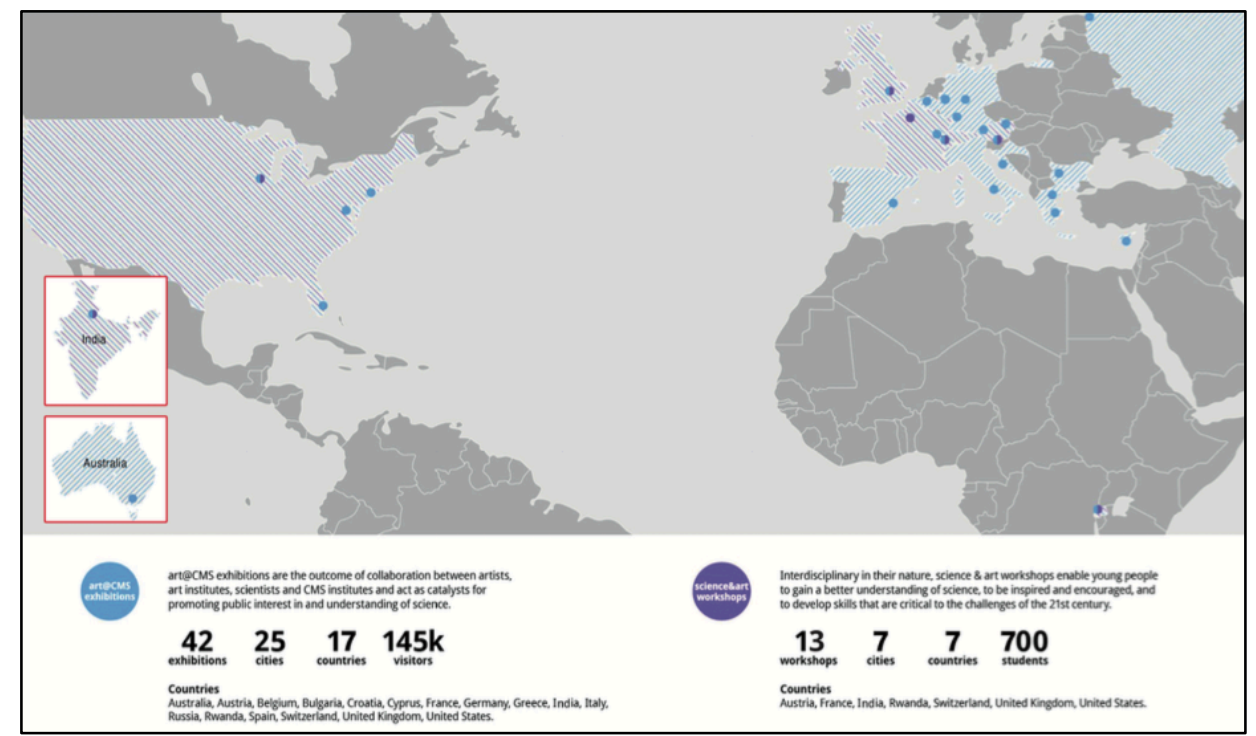

Fig 3: Geographical map of the 42 art@CMS exhibitions and 13 workshops across the world.

\subsubsection{SciArt workshops}

SciArt workshops are cross-disciplinary learning activities designed to introduce school, college and university art and science students to the scientific world of particle physics and fundamental research through artistic inquiry and creation. They incorporate an introduction to particle physics, CMS and CERN, followed by philosophical reflection and discussion aimed at inspiring the students to develop their own science-related art works. The students then have the opportunity to mount an exhibition of their work.

These STEAM workshops foster skills that are more appropriate to the challenges of the 21 st century. Much work has already been done to quantify the benefits of developing skills such as creativity, critical thinking and collaboration [24]. Aiming to act like an ideas factory, art@CMS SciArt workshops are built on a platform for learning, sharing and sustainable personal development. They are ultimately a form of learner(s) hub where the individual can take ownership of their ideas, and turn those ideas into tangible products of imaginative thinking that embody both aesthetic and educational value [25]. Since 2013, 13 SciArt workshops have taken place in seven countries engaging 700 school, college and university students. Two examples of such workshops are presented briefly below. 

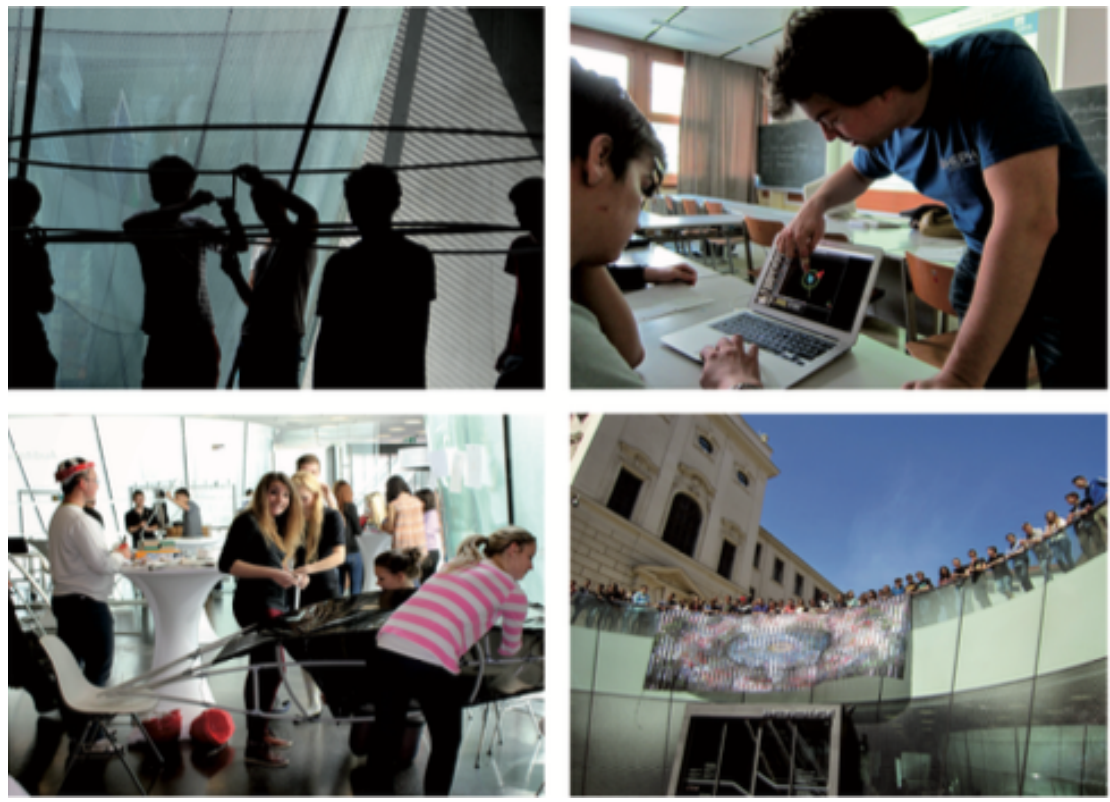

Fig. 4. Students at work during the art@CMS SciArtworkhop in Graz, Austria

\subsubsection{High energy physics meets art (Graz, Austria, June 2013)}

The first in the series of art@CMS SciArt workshops took place in Graz, Austria, with the participation of 62 high-school students from two local schools: GIBS and BORG. This threeday workshop, as shown in Figure 4, was supported by CMS, the Institute for High Energy Physics of the Austrian Academy of Science (HEPHY) with the collaboration of the National History Museum in Graz and the City of Graz.

On the first day, HEPHY physicists delivered a Masterclass where the students learned to visualise and analyse real LHC data from the CMS experiment. The students were then introduced to the interconnections of science and the arts by learning how artists visualise science and technology in their works.

On the second day, four groups of students under the guidance of art educators and scientists, created their own art works inspired by particle physics. A separate group of students documented the whole workshop with photos, a video and a dedicated blog. On the last day, the art works were displayed for public viewing at Graz's National History Museum attracting 200 visitors, including parents and other students.

\subsubsection{Imagining physics: art inspired by Fermilab (Batavia, US, February 2015)}

Through a collaboration between Fermilab, Water Street Studios, local artists and highschool students, a SciArt workshop, entitled "Imaging Physics" was organized in Batavia, US, in February 2015 as part of an art@CMS exhibition hosted by Fermilab Art Gallery. The workshop brought together 18 high school art students with the aim to introduce them into the world particle physics and to challenge them to turn their impressions into art. 
Over the course of two weeks, the students worked collaboratively under the guidance of their teachers and professional artists from Water Street Studios to create artworks inspired by particle physics. They first visited Fermilab where they took part in presentations by physicists and also learned about art@CMS from Michael Hoch. Four hands-on meetings were then organised in Water Street Studios during which the students worked on their ideas by developing art works that encapsulated how particle physics connect to their own lives. The art works were then transferred to Fermilab Art Gallery for public viewing.

"Having the Art@CMS pieces here at Fermilab is outstanding," said Georgia Schwender, curator of the Fermilab Art Gallery. "But having the chance to connect the art and science of the CMS experiment with students outside the laboratory makes this event a true example of our mission." [26]

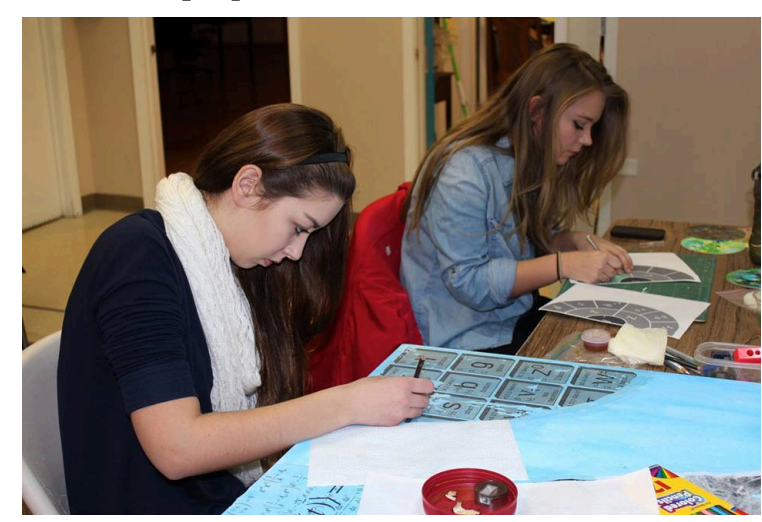

Figure 5a.art@CMS SciArtworkshop in Water Street Studios, Batavia, US.

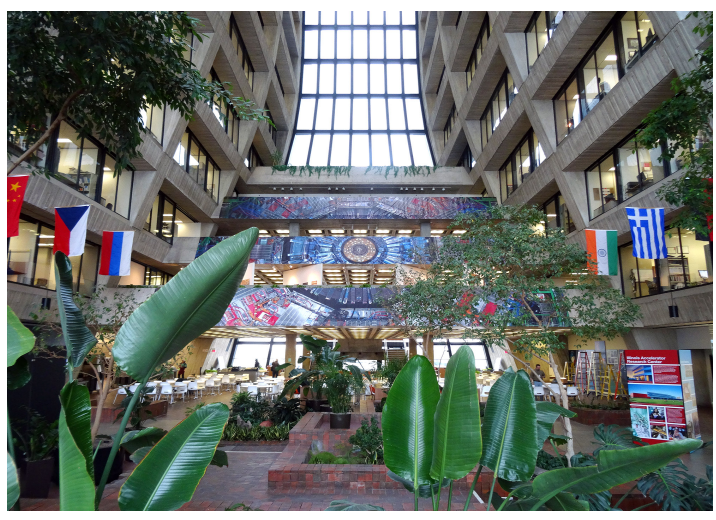

Figure 5b. General view of the art@CMS exhibition in Fermilab's Art Gallery.

\section{4."Art and Science across Italy"}

The "Art and Science across Italy" project has been developed by the INFN in collaboration with the CMS Communications group and with the support of the CREATIONS EU project. The principal goal of the project is to act as an education and outreach bridge between school and teacher communities in Italy and scientists in high-energy physics through the organization of STEAM activities including SciArt exhibitions and workshops across four cities: Milano, Padova/Venice, Florence and Naples. The project consists of three stages as follows.

The first stage includes the scientific part. Through seminars, meetings and visits to INFN laboratories organized by project's coordination committee, selected students from various high schools (e.g., classic, science, art) will have the opportunity to interact with INFN researchers and understand how science and technology works in high-energy physics.

The second stage incudes the creative part. Cross-disciplinary student teams, with no more than four students per team, will be formed with the aim to work collaboratively for the creation of art works inspired by what they learned during the first stage. The teams will be encouraged to work with any art medium they consider appropriate to turn their ideas into creative and engaging outcomes inspired by particle physics. 
In the last stage, the best art works will be selected by the coordination committee with the help of art experts of the Italian science museums involved in the project, including the Leonardo Da Vinci museum in Milano and Città della Science museum in Napoli. The selection will be at a local and national level as follows. The best local-level art works will be incorporated into the "I colori del Bosone di Higgs" traveling exhibition across the four aforementioned cities. At a national level, two student art artworks will be selected among the local art works. The two winning student teams will be invited to visit CERN, while their art works will be included in the art@CMS collection and be shown in the art@CMS traveling exhibitions.

The "Science and Art in Italy" project will commence in March 2017 in Milano and will then continue to Venice for the EPS-HEP 2017 conference in July 2017, followed by Florence in November 2017. The final destination will be Naples in spring 2018, where a summative even will take place with the participation of students, teachers, scientists and the organizers.

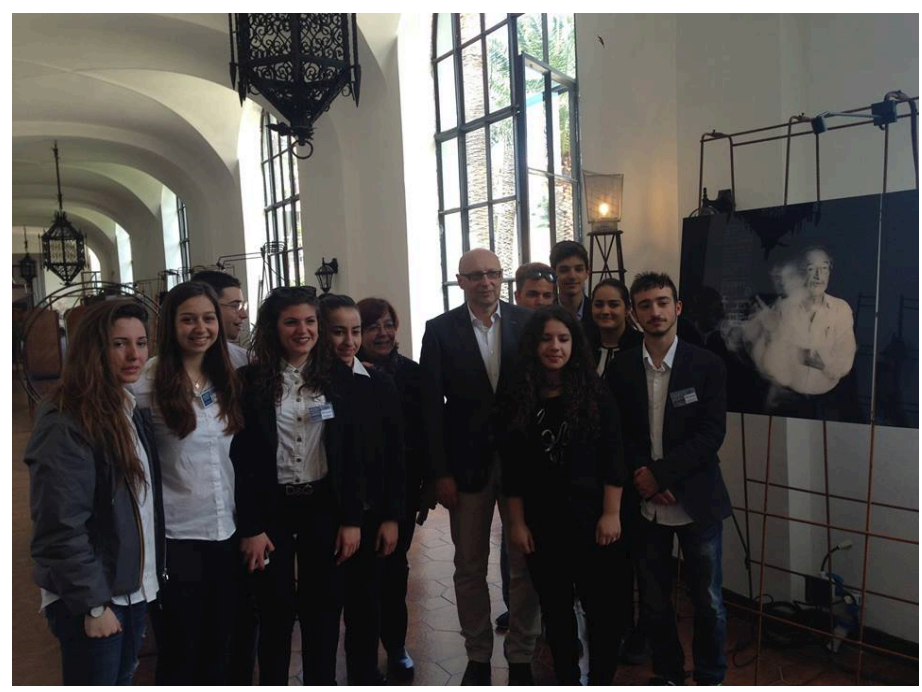

Fig 6: Nobel price Prof. Stefan Hell visits the "Arte \& Scienza" exhibition held in Napoli (May 2016). Lyceum students were the guides of the exhibition after having had some meetings and seminars with INFN researchers and professors.

The project has been piloted successfully in April 2016 in Vico Equense, Naples, during the "premio Capo d'Orlando" event. Students from twelve local schools and about 2,500 members of the general public visited the art@CMS exhibition that was organized by the CMS experiment and INFN, Napoli, with the support of the scientific Lyceum of Sorrento, which provided more than fifteen students who were trained to act as exhibition guides by INFN researchers and their physics school teachers.

\section{Conclusion}

This paper presented a set of novel education and outreach activities by the ATLAS and CMS collaborations that fall into the STEAM movement. Despite some differences, all of these 
activities, taken collectively, can play a transformative role in enabling high-energy physics to become accessible to larger and more diverse audiences while also encouraging the development of $21^{\text {st }}$ century skills to the next generation of learners regardless if they decide to follow scientific careers or not.

\section{Acknowledgements}

On behalf of CMS, we would like to acknowledge the following people for their contribution to the art@CMS activities presented in this paper: Mick Storr (CERN/University of Birmingham), Stephen Preece (All Minds and ECOLINT) and Achille Petrilli (CERN).

The art@CMS activities presented in this paper are partially funded by the European Union in the context of the CREATIONS project (Grant Agreement no. 665917) under the "Science with and for Society" section of the Horizon 2020 Work Programme. This document does not represent the opinion of the European Union, and the European Union is not responsible for any use that might be made of its content.

On behalf of ATLAS, we would like to acknowledge the following people who are involved in the two ATLAS projects which are further documented in [13] and [18]: many thanks to Steven Gordfarb (University of Melbourne, Australia), Ewan Hill (University of Victoria, TRIUMF, Canada), Juliana Cherston and Joe Paradiso (MIT Media Lab), Agnes Chavez (artist, STEMarts Lab), Markus Dorninger (artist, Tagtool App, OMAI), Luis Flores Castillo (Chinese University of Hong Kong) and Michael Wadness (educator, Quarknet fellow).

\section{References}

[1] Y. Hadzigeorgiou, Imaginative science education, Springer, 2016.

[2] Americans for the Arts, Arts education navigator: facts \& figures, 2013. Retrieved from http://www.americansforthearts.org/by-program/networks-and-councils/arts-educationnetwork/tools-resources/arts-ed-navigator/facts-figures

[3] C. Ryan et al., Science education for responsible citizenship, European Commission, 2015.

[4] Y. Hadzigeorgiou, N. Fotinos, Imaginative thinking and the learning of science, Science Education Review 6 (2007).

[5] J. Osborne et al., What "ideas-about-science" should be taught at school? A Delphi study of the expert community, Journal of Research in Science Teaching 40 (2003).

[6] K. Robinson, Do schools kill creativity? (video), 2006. Retrieved from http://www.ted.com/index.php/talks/view/id/66

[7] J. Graff, Practitioner guide. The nature of learning: Using research to inspire practice, OECD, 2012.

[8] U. Billow et al., Resources for E\&O, in proceedings of EPS-HEP conference, PoS(EPSHEP2015)619.

[9] http://www.stemartslab.com/project/projecting-particles-workshop/ 
[10] http://www.omai.at/tagtool/

[11] http://atlas-live-virtual-visit.web.cern.ch/atlas-live-virtual-visit/

[12] http://atlas.cern/updates/atlas-news/projecting-particles-cuba

[13] https://cds.cern.ch/record/2201662/files/ATL-OREACH-SLIDE-2016-427.pdf

[14] https://www.media.mit.edu/research/groups/responsive-environments

[15] http://quantizer.media.mit.edu/

[16] http://atlas.cern/updates/atlas-news/musical-dimensions

[17] http://dl.acm.org/citation.cfm?id=2892295

[18] https://cds.cern.ch/record/2201663/files/ATL-OREACH-SLIDE-2016-428.pdf

[19] http://cms.web.cern.ch

[20] M. Hoch, A. Alexopoulos, Art@CMS and Science\&Art@School: Novel education and communication channels for particle physics, DOI: http://dx.doi.org/10.1142/9789814603164_0115

[21] http://artcms.web.cern.ch/artcms/

[22] M. Hoch et al., CMS - The art of science, Edition Lammerhuber, 2016.

[23] http://creations-project.eu

[24] R. Paul, L. Elder, A guide for education to critical thinking competency standards: Standards, principles, performance, Foundation for Critical Thinking, 2005.

[25] http://cylindricalonion.web.cern.ch/blog/201509/science-and-art-combine-international-schoolgeneva

[26] http://news.fnal.gov/2015/01/arts-extravaganza-at-fermilab-to-celebrate-one-of-the-worlds-largestscience-experiments/

[27] https://www.youtube.com/watch?v=Grjncr66xhY 